\title{
Texture Feature Extraction of RGB, HSV, YIQ and Dithered Images using Wavelet and DCT Decomposition Techniques
}

\author{
Er. Manisha Lumb \\ Research Scholar, D.A.V.I.E.T, Jalandhar
}

\author{
Er. Poonam Sethi \\ Asst. Prof. D.A.V.I.E.T, Jalandhar
}

\begin{abstract}
An image can be retrieved from number of features contained in it. But it depends upon its format, which features are best selected for the proper retrieval. In this paper, the RGB, HSV, YIQ and dithered images are retrieved using two computational retrieval techniques; DCT and Wavelet decomposition. When used DCT transformation technique, only HSV images are giving the best results, while when Wavelet transformation is used, the HSV, Dithered and YIQ images are giving satisfactory results, out of which from the accuracy point of view, HSV images are having maximum degree of accuracy in correct retrieval. After analysis, it is found that in DCT as well as in Wavelet decomposition techniques, the HSV images are correctly retrieved.
\end{abstract}

\section{KEYWORDS}

HSV (Hue Saturation Value), YIQ (NTSC luminance $(Y)$ and chrominance ( $I$ and $Q$ ) color components)

\section{INTRODUCTION}

Recently, with the advances in various multimedia technologies, such as high speed network, compression and new digital image sensor technologies, large image databases are being created by scientific, educational, medical, industrial and other applications. These large volumes of the images make difficult for a user to browse through the entire database. Therefore, an efficient and automatic procedure is a need for indexing and retrieving images from databases [1].

With the development of the Internet, and the availability in image capturing devices such as digital cameras, image scanners, the size of digital image collection is increasing rapidly. It is very important to efficiently store and retrieve images for different application such as fashion design, crime prevention, medicine, architecture, etc [2].

\subsection{Content Based Image Retrieval (CBIR)}

There are two approaches to image retrieval: Text-Based approach and Content- Based approach. Today, the most common way of doing this is by textual descriptions and categorizing of images. This approach has some obvious shortcomings. Different people might categorize or describe the same image differently, leading to problems retrieving it again. It is also time consuming when dealing with very large databases. Content based image retrieval (CBIR) is a way to get around these problems. CBIR systems search collection of images based on features that can be extracted from the image files themselves without manual descriptive. In past decades many CBIR systems have been developed, the common ground for them is to extract a desired image. Comparing two images and deciding if they are similar or not is a relatively easy thing to do for a human. Getting a computer to do the same thing effectively is however a different matter [3].

CBIR is a technique which uses visual content to search and compare images from large scale image databases according to the interests of users. In such databases each image is represented by a unique signature. Visual content of the image like color, shape, texture and spatial layout are widely used in CBIR [4].

The algorithms used in these systems are commonly divided into three tasks:

- extraction
- selection and
- classification

The extraction task transforms rich content of images into various content features. Feature extraction is the process of generating features to be used in the selection and classification tasks. Feature selection reduces the number of features provided to the classification task. Those features which are likely to assist in discrimination are selected and used in the classification task. Features which are not selected are discarded [5].

The major issue in texture analysis is to compute the characteristic of a digital image that is able to numerically describe its texture properties. [6]

\section{FEATURE EXTRACTION OF IMAGES USING DIFFERENT FORMATS}

\subsection{Feature extraction in RGB}

Basic idea behind these features is to split an image into tiles called cells and then extract a weighted histogram of gradient orientations for each cell. Defining multiple resolutions, Gradient computation and computing histogram of gradient orientations are the steps in feature extraction [7]. $R G B$ colours are called primary colours 
and are additive. By varying their combinations, other colours can be obtained [5]. The image given below is the RGB image which is to be extracted from the database available.

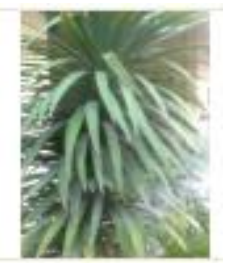

Fig.1 RGB Query image

\subsection{Feature extraction in HSV}

It is essential to quantify HSV space component to reduce computation and improve efficiency. Unequal interval quantization according to the human color perception has been applied on $\mathrm{H}, \mathrm{S}$ and $\mathrm{V}$ components. In accordance with the different colors and subjective color perception quantification, quantified hue $(\mathrm{H})$, saturation (S) and intensity (V) are obtained [8]. Hue ranges from 0 to 360 degrees, with variation beginning with red, going through yellow, green, cyan, blue and magenta and back to red [5].

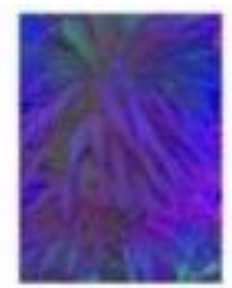

Fig.2 HSV image

\subsection{Feature Extraction in YIQ}

For a color image, the RGB model is the well-known color model. However, it is not a uniform color model. The YIQ model is more related to the human perception, since it can isolate luminance and chrominance. Hence, in this paper, the image retrieval is performed using YIQ model. Because a probability distribution is uniquely characterized by its moments, the color distributions of the Y, I, and Q components of an image can be represented respectively by its color moments. The first color moment of the $i$-th color component $(i=1,2,3)$ is defined by

$$
M_{i}^{1}=\frac{1}{N} \sum_{j=1}^{N} P_{i, j}
$$

Where $\boldsymbol{P}_{i, j}$, is the color value of an $i$-th color component of the $j$-th image pixel and $N$ is the total number of pixels in the image.
The $Y I Q$ color space is adopted by the NTSC (National Television System Committee) video standard in reference to $R G B N T S C$ [9]. The color space of YIQ images is given by:

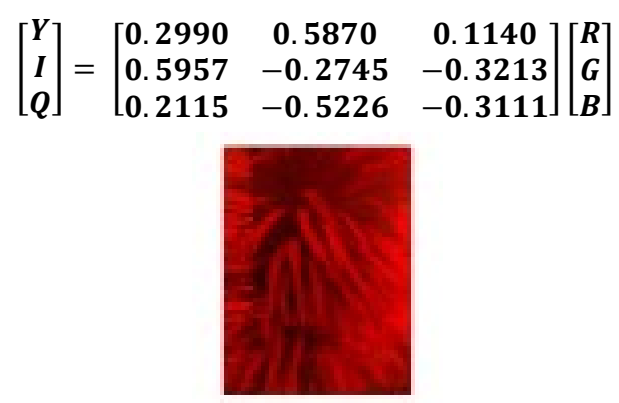

Fig. 3 YIQ image

\subsection{Texture Feature extraction from the dithered image}

Image dithering is often used for commercial print products like newspapers and magazines. Many printers (e.g. laser and inkjet printers) can only put a series of single black dots on paper. Intensity is then regulated by the local density of the dots. This process is called dithering. However, when a dithered image is scanned, the interaction between the discrete scanning process and dithering may seriously degrade the image. Dithering and scanning are often both periodic, leading to periodic interference artifacts. These can be removed by proper filtering in the spatial 2D Fourier domain. It is simplest to replace each pixel with a small array of random black dots, with the number of dots proportional to the intensity. [10]

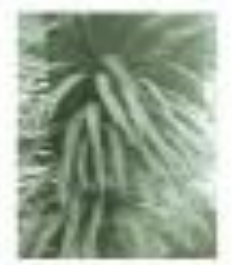

Fig.4 Dithered image

\section{TECHNIQUES FOR FEATURE EXTRACTION \\ 3.1 Use of Wavelet transformation for feature extraction:}

The wavelet transform is a multi-resolution technique, which can be implemented as a pyramid or tree structure and is similar to sub-band decomposition. There are various wavelet transforms like Haar, Daubechies, Coiflet, Symlet and etc. They differ with each other in the formation and reconstruction. The wavelet transform divides the original image into four subbands and they are denoted by LL(lowlow), LH(low-high), HL(highlow) and $\mathrm{HH}$ (high-high) frequency subbands. The $\mathrm{HH}$ subimage represents diagonal details (high frequencies in both directions - the corners), HL gives horizontal high frequencies (vertical edges), LH gives vertical high frequencies (horizontal edges), and the image LL corresponds to the lowest frequencies. At the subsequent 
scale of analysis, the image LL undergoes the decomposition using the same filters, having always the lowest frequency component located in the upper left corner of the image. Each stage of the analysis produces next 4 sub images whose size is reduced twice when compared to the previous scale. i.e. for level ' $n$ ' a total of ' $4+(\mathrm{n}-1)^{*} 3$ ' sub bands are obtained. The size of the wavelet representation is the same as the size of the original. The Haar wavelet is the first known wavelet and was proposed in 1909 by Alfred Haar. Haar used these functions to give an example of a countable orthonormal system for the space of square -integrable functions on the real line [15].

\subsection{Feature extraction using DCT:}

In general, neighboring pixels within an image tend to be highly correlated. As such, it is desired to use an invertible transform to concentrate randomness into fewer, de-correlated parameters. The Discrete Cosine Transform (DCT) has been shown to be near optimal for a large class of images in energy concentration and decorrelating. It has been adopted in the JPEG and MPEG coding standards [12].

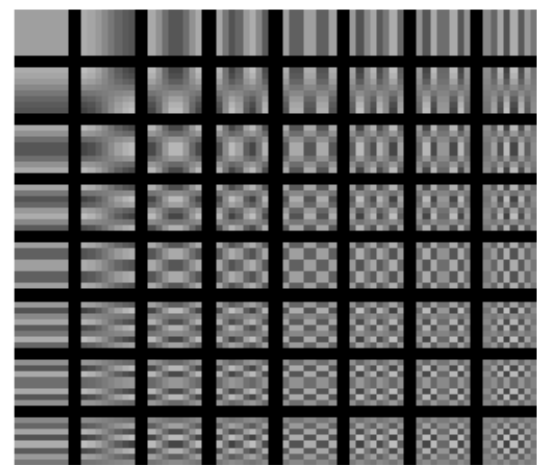

Fig 5. The 64 Basis Functions of an 8-by-8 Matrix

Horizontal frequencies increase from left to right, and vertical frequencies increase from top to bottom. The constant-valued basis function at the upper left is often called the DC basis function, and the corresponding DCT coefficient is often called the DC coefficient.

The DCT decomposes the signal into underlying spatial frequencies, which then allow further processing techniques to reduce the precision of the DCT coefficients consistent with the Human Visual System (HVS) model. The DCT coefficients of an image tend themselves as a new feature, which have the ability to represent the regularity, complexity and some texture features of an image and it can be directly applied to image data in the compressed domain. This may be a way to solve the large storage space problem and the computational complexity of the existing methods [12]. In contrast, discrete cosine transform (DCT) of real data avoids complex arithmetic and offers ease of implementation in practical applications. Moreover, DCT can efficiently handle the phase unwrapping problem and exhibits a strong energy compaction property, i.e., most of the signal information tends to be concentrated in a few low components of the DCT [13].

\section{EXPERIMENT AND ANALYSIS}

In this paper, the images that are similar to the query image of the plant as shown below is retrieved from the set of images. There is a set of 500 images including the HSV, YIQ and dithered images from which the query image is retrieved successively.

There is a set of RGB image database given below through which the images which are similar to the query image are retrieved:

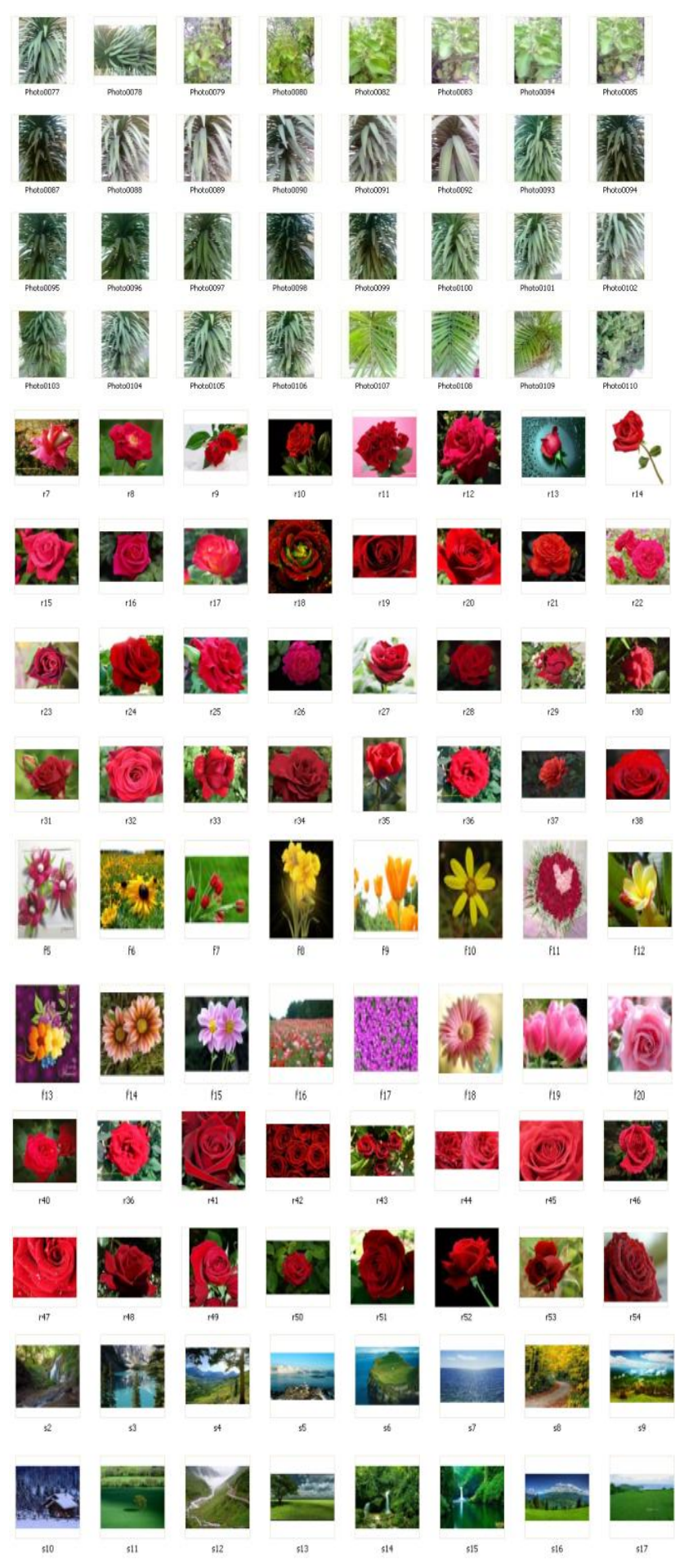

Fig. 6 Data base of different set of images. 
Firstly, the RGB, HSV, YIQ and Dithered images are computed through Wavelet transformation technique, and after this DCT transformation will be used for image retrieval. The following results are obtained from above computational techniques.

\subsection{Feature extraction using Discrete wavelet transform (DWT):}

Now, Wavelet decomposition technique will be used for extracting the similar images. In the wavelet decomposition technique, the parameters; vertical component, horizontal component, standard deviation and mean are calculated from the approximation coefficients matrix cA of the wavelet transformed image which is obtained through Wavelet transforming of an image. The wavelet transformation performs single-level two-dimensional wavelet decomposition with respect to a particular wavelet (Haar). It computes the approximation coefficients matrix $\mathrm{cA}$ and details coefficients matrices $\mathrm{cH}, \mathrm{cV}$, and $\mathrm{cD}$ (horizontal, vertical, and diagonal, respectively), obtained by wavelet decomposition of the input matrix (Query mage).

\subsubsection{For RGB images:}

Table 1 Wavelet decomposition parameter for RGB image

\begin{tabular}{|l|l|}
\hline Name of the image & Vertical Component \\
\hline Query Image & 1.3917 \\
\hline 80 & 1.3908 \\
\hline 87 & 1.3949 \\
\hline 89 & 1.3934 \\
\hline 91 & 1.3902 \\
\hline 94 & 1.3941 \\
\hline 97 & 1.3938 \\
\hline 98 & 1.3980 \\
\hline 99 & 1.3903 \\
\hline 100 & 1.3927 \\
\hline
\end{tabular}

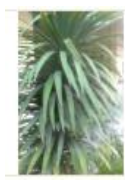

RGB query image
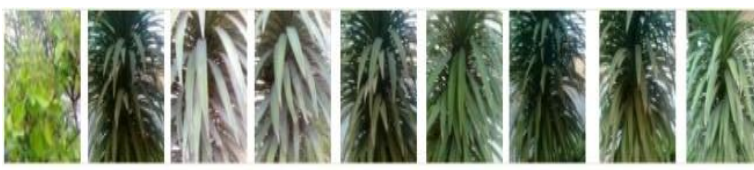

Fig.7 Retrieved RGB images using Wavelet Decomposition 
The RGB images retrieved using Wavelet Decomposition is shown above. From the above result, it can be seen that the RGB images can be retrieved on the basis of vertical component of wavelet transformed image. 9 images are retrieved, out of which 8 are the correct one. Thus the accuracy of correct retrieval is $89 \%$.

\subsubsection{For HSV images:}

Table 2: Wavelet decomposition parameter for HSV image

\begin{tabular}{|l|l|l|l|l|}
\hline $\begin{array}{l}\text { Name } \\
\text { of the } \\
\text { image }\end{array}$ & Mean & $\begin{array}{l}\text { Standard } \\
\text { Deviation }\end{array}$ & $\begin{array}{l}\text { Horizontal } \\
\text { Component }\end{array}$ & $\begin{array}{l}\text { Vertical } \\
\text { Component }\end{array}$ \\
\hline $\begin{array}{l}\text { Query } \\
\text { Image }\end{array}$ & 288.8895 & 181.4355 & 1.0579 & 1.0596 \\
\hline 88 & 281.5365 & 187.3006 & - & 1.0557 \\
\hline 89 & - & 189.3897 & 1.0595 & 1.0517 \\
\hline 90 & 281.8535 & 187.2095 & - & 1.0535 \\
\hline 91 & - & - & 1.0583 & - \\
\hline 92 & 281.6056 & 187.7221 & - & 1.0587 \\
\hline 93 & - & - & 1.0532 & 1.0514 \\
\hline 95 & 287.2069 & 182.6033 & - & - \\
\hline 97 & 289.2069 & 181.3430 & - & - \\
\hline 99 & - & 180.3994 & - & - \\
\hline 100 & - & - & 1.0574 & 1.0562 \\
\hline 101 & - & - & 1.0520 & 1.0579 \\
\hline 102 & 283.1772 & 186.1202 & 1.0576 & 1.0580 \\
\hline 103 & 287.6047 & 182.5818 & - & - \\
\hline 104 & 287.7214 & 182.4500 & - & - \\
\hline 105 & 289.1675 & 181.2741 & 1.0588 & 1.0516 \\
\hline 106 & 289.5398 & 181.5731 & - & - \\
\hline 108 & - & - & 1.0558 & 1.0556 \\
\hline & & & & \\
\hline
\end{tabular}

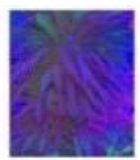

HSV Query image

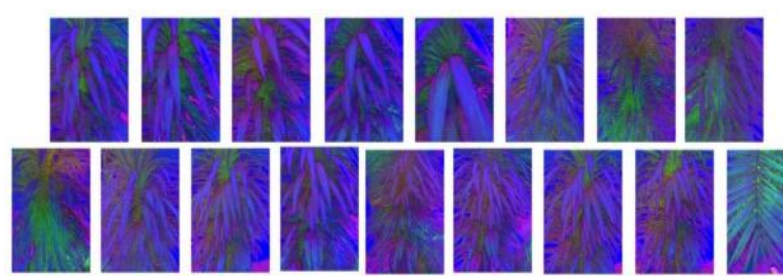

Fig.8 Retrieved HSV images using Wavelet Decomposition
Through Wavelet Decomposition, the above similar images are retrieved on the basis of mean, standard deviation, horizontal and vertical component of the decomposed image. It can be seen that all retrieved images are similar to the query image. RGB is an m-byn-by-3 image array whose three planes contain the red, green, and blue components for the image. HSV is returned as an m-by-n-by-3 image array whose three planes contain the hue, saturation, and value components for the image. Out of the above 17 retrieved images, 16 are of the correct one and only one image is of different plant. Thus the accuracy of correct retrieval is $94 \%$.

Wavelets are functions generated from one single function $\psi$ by dilations and translations. The basic idea of the wavelet transform is to represent any arbitrary function as a superposition of wavelets. Any such superposition decomposes the given function into different scale levels where each level is further decomposed with a resolution adapted to that level.

\subsubsection{For dithered Images:}

Table 3 Wavelet decomposition parameter for Dithered image

\begin{tabular}{|c|c|c|}
\hline $\begin{array}{l}\text { Name of } \\
\text { the image }\end{array}$ & $\begin{array}{l}\text { Standard } \\
\text { Deviation }\end{array}$ & $\begin{array}{l}\text { Horizontal } \\
\text { Component }\end{array}$ \\
\hline $\begin{array}{l}\text { Query } \\
\text { Image }\end{array}$ & 127.5445 & 0.7987 \\
\hline 6 & 124.6003 & 0.7975 \\
\hline 7 & - & 0.7917 \\
\hline 10 & 125.2246 & - \\
\hline 13 & 125.7092 & - \\
\hline 16 & - & 0.7937 \\
\hline 17 & - & 0.7925 \\
\hline 18 & - & 0.7983 \\
\hline 19 & 127.5259 & 0.7963 \\
\hline 20 & 128.7653 & - \\
\hline 21 & 120.3331 & - \\
\hline 24 & 125.9096 & - \\
\hline 32 & - & 0.7942 \\
\hline 33 & - & 0.7958 \\
\hline 35 & 124.5738 & 0.7940 \\
\hline
\end{tabular}

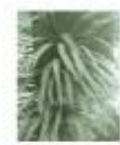

Dithered Query image 

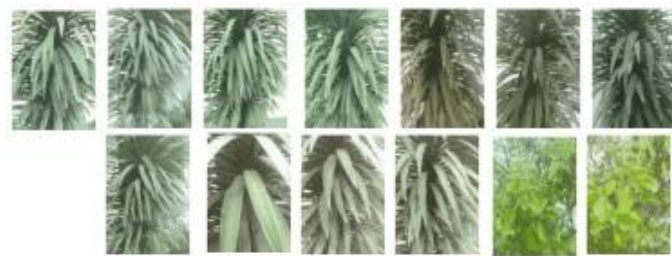

Fig.9 Retrieved Dithered images using Wavelet Decomposition

Out of the images in the data base, the above are retrieved after wavelet decomposition of dithered images. Here also, almost the images are similar to the query image. These images are retrieved on the basis of standard deviation and horizontal component. Out of 14 images retrieved 12 are the correct one. Last two images are of different plant. Thus the accuracy of correct retrieval can be calculated by the ratio of correctly retrieved images upon total images retrieved i.e $86 \%$.

\subsubsection{For YIQ images:}

Now for YIQ images, the wavelet transform of the images are conducted and then required parameters are calculated to retrieve the correct images.

Table 4 Wavelet decomposition parameter for YIQ image

\begin{tabular}{|l|l|l|}
\hline $\begin{array}{l}\text { Name of the } \\
\text { image }\end{array}$ & $\begin{array}{l}\text { Horizontal } \\
\text { Component }\end{array}$ & $\begin{array}{l}\text { Vertical } \\
\text { Component }\end{array}$ \\
\hline Query Image & 0.7800 & 0.8025 \\
\hline 77 & - & 0.8194 \\
\hline 83 & 0.7835 & - \\
\hline 85 & - & 0.8056 \\
\hline 87 & 0.7932 & - \\
\hline 93 & 0.7784 & - \\
\hline 94 & 0.7732 & - \\
\hline 95 & 0.7956 & 0.8279 \\
\hline 96 & 0.7843 & - \\
\hline 98 & - & 0.8010 \\
\hline 100 & - & 0.8294 \\
\hline 101 & - & 0.8218 \\
\hline 103 & 0.7982 & 0.8303 \\
\hline 104 & 0.7956 & 0.8390 \\
\hline 105 & - & 0.8301 \\
\hline 106 & 0.7617 & 0.8359 \\
\hline 108 & 0.7882 & - \\
\hline
\end{tabular}

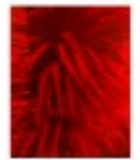

YIQ Query Image
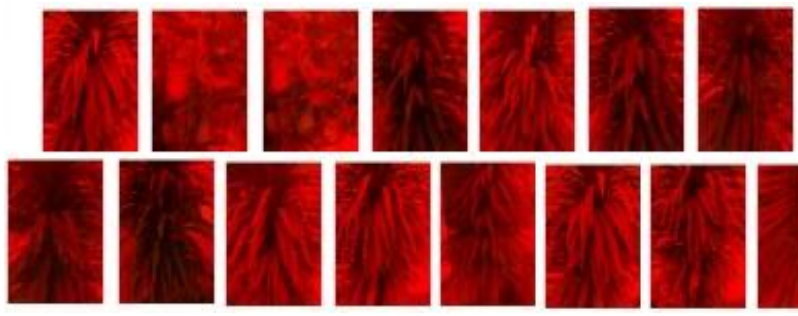

Fig.10 Retrieved YIQ images using Wavelet Decomposition

From the above result of image retrieval, it can be seen that the YIQ images can easily retrieved on basis of horizontal and vertical component of wavelet transformed images. 15 images are retrieved, out of which 12 are the correct one. Hence the percentage accuracy of correct retrieval is $80 \%$.

\subsection{Feature extraction using DCT:}

Now days, DCT is adopted by many compression standards such as JPEG, MPEG 1/2, H.261/H.263. The standard JPEG defined by the Joint Picture Expert Group is a commonly used method for the loss image compression for photographic images. The JPEG compression is a transform-based coding algorithm [17].

\subsubsection{DCT for RGB:}

Table 5. DCT parameter for RGB image

\begin{tabular}{|l|l|}
\hline $\begin{array}{l}\text { Name of the } \\
\text { image }\end{array}$ & Mean \\
\hline Query Image & 0.1020 \\
\hline 92 & 0.1076 \\
\hline 96 & 0.1078 \\
\hline 101 & 0.1008 \\
\hline 105 & 0.1052 \\
\hline 108 & 0.1049 \\
\hline 109 & 0.1054 \\
\hline
\end{tabular}

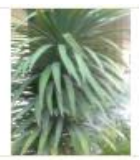

RGB query image
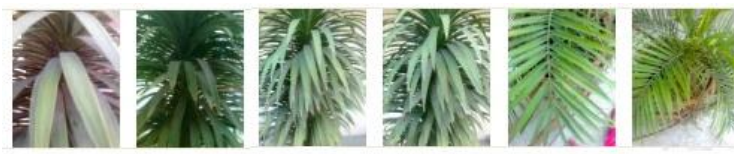

Fig.11 Retrieved RGB images using DCT Transform 
The images shown in fig.14 are retrieved RGB images when DCT transformation is used. Here query image is also given at the top. It can be seen that out of 6 retrieved images, only 4 are the correct one. Thus the accuracy for correct retrieval is ratio number of correctly retrieved images to the total retrieved images i.e. $67 \%$.

\subsubsection{DCT For HSV}

Now, DCT transformation of HSV images is also done. After the transformation, different parameters are calculated, on the basis of which HSV images are giving the best performance for DCT transformation technique.

Table 6. DCT parameter for HSV image

\begin{tabular}{|c|c|}
\hline $\begin{array}{l}\text { Name of the } \\
\text { image }\end{array}$ & Correlation Co-efficient \\
\hline 87 & 0.9933 \\
\hline 88 & 0.9953 \\
\hline 89 & 0.9947 \\
\hline 90 & 0.9951 \\
\hline 91 & 0.9945 \\
\hline 92 & 0.9949 \\
\hline 93 & 0.9952 \\
\hline 94 & 0.9947 \\
\hline 95 & 0.9944 \\
\hline 96 & 0.9943 \\
\hline 97 & 0.9952 \\
\hline 98 & 0.9922 \\
\hline 99 & 0.9942 \\
\hline 100 & 0.9954 \\
\hline 101 & 0.9953 \\
\hline 102 & 0.9956 \\
\hline 103 & 0.9952 \\
\hline 104 & 0.9954 \\
\hline 105 & 0.9957 \\
\hline 106 & 0.9947 \\
\hline
\end{tabular}

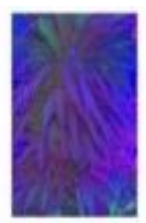

Query image
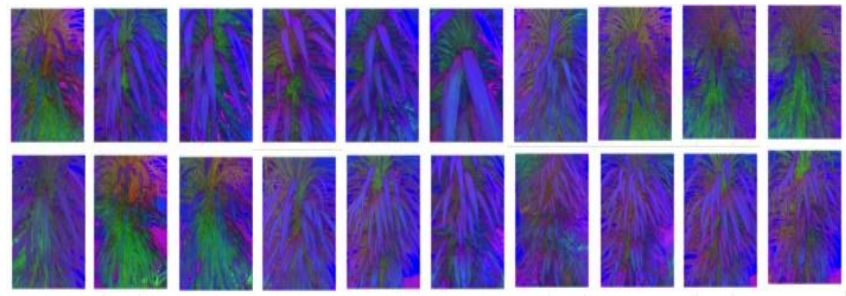

Fig.12 Retrieved HSV images using DCT Transform

The above figure shows that 20 images are retrieved correctly from the database. Thus the accuracy of correct retrieval, which is equal to the ratio of number of correct images retrieved to the total number of images retrieved, is equal to $100 \%$.

\subsubsection{DCT for Dithered Images}

Table 7. DCT parameter for Dithered image

\begin{tabular}{|l|l|}
\hline Name of the image & Entropy \\
\hline Query Image & 1.5012 \\
& \\
\hline Deth_8 & 1.5023 \\
\hline Deth_15 & 1.5752 \\
\hline Deth_17 & 1.5325 \\
\hline Deth_16 & 1.5707 \\
\hline Deth_20 & 1.5127 \\
\hline Deth_25 & 1.5674 \\
\hline
\end{tabular}

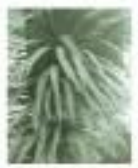

Query Dithered Image

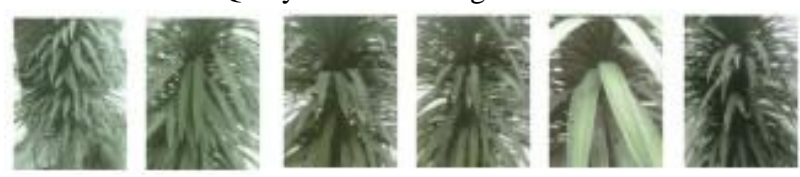

Fig.13 Retrieved Dithered images using DCT

\section{Transform}

The above images are retrieved images using DCT transformation, in which it can be seen that all the images are correct. Thus accuracy of correct retrieval is $100 \%$. 


\subsubsection{DCT For YIQ}

Table 8. DCT parameter for YIQ image

\begin{tabular}{|c|c|c|}
\hline $\begin{array}{c}\text { Name of the } \\
\text { image }\end{array}$ & Mean & Entropy \\
\hline Query Image & 0.2383 & 4.0559 \\
\hline 77 & 0.2383 & \\
\hline 88 & 0.2383 & \\
\hline 89 & 0.2383 & \\
\hline 92 & 0.2383 & \\
\hline 93 & 0.2383 & \\
\hline 96 & - & 4.0142 \\
\hline 97 & - & 4.0603 \\
\hline 100 & 0.2383 & 4.0125 \\
\hline 101 & 0.2383 & \\
\hline 105 & 0.2383 & \\
\hline 106 & 0.2383 & \\
\hline 109 & - & 4.0349 \\
\hline 110 & 0.2383 & \\
\hline
\end{tabular}
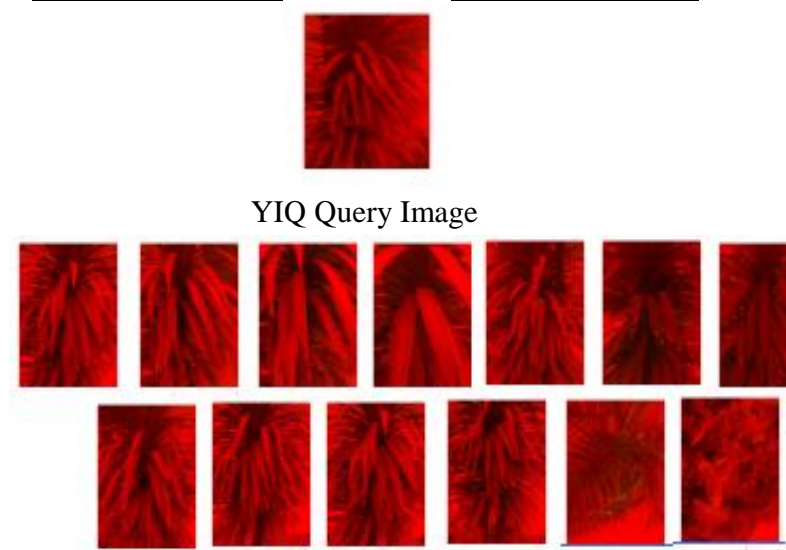

Fig.17 Retrieved YIQ images using DCT Transform

As it can be seen that total 13 images are retrieved, out of which 11 are the correct one. Thus the accuracy of correct retrieval is given by $85 \%$.

\subsection{Combining the result of Accuracy in correct retrieval} and accuracy rate

\subsubsection{Accuracy in correct retrieval:}

From the following details of the accuracy in correct retrieval, the accuracy in retrieval of different images in different formats can be easily distinguished. The most important thing is in correctness in retrieval of images which is covered in this section.
The table below is showing Accuracy in correct retrieval for all the four formats (RGB. HSV, YIQ and Dithered) using both techniques i.e. DWT and DCT.

Table 9. Accuracy in DWT and DCT computation

\begin{tabular}{|c|c|c|}
\hline Format & $\begin{array}{c}\text { Accuracy with } \\
\text { DWT }\end{array}$ & $\begin{array}{c}\text { Accuracy with } \\
\text { DCT }\end{array}$ \\
\hline RGB & $89 \%$ & $67 \%$ \\
\hline HSV & $94 \%$ & $100 \%$ \\
\hline YIQ & $80 \%$ & $85 \%$ \\
\hline Dithered & $86 \%$ & $100 \%$ \\
\hline
\end{tabular}

Graph 1. Accuracy in correct retrieval

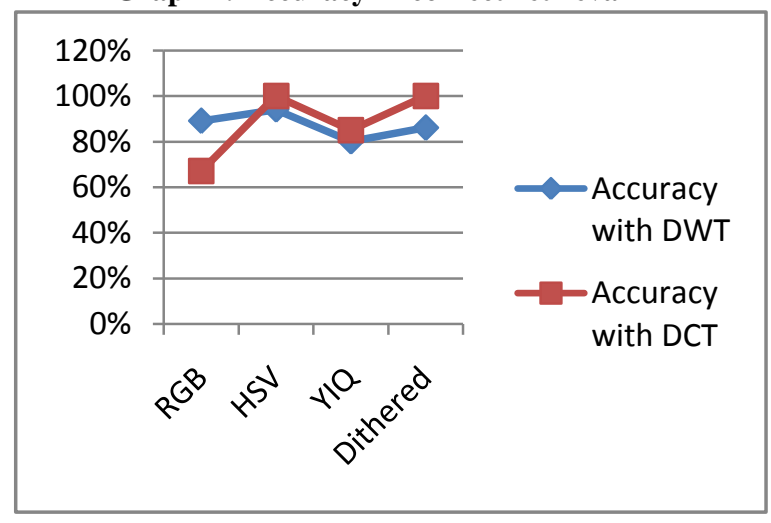

From the above graph, it can be clearly explored that out of the retrieved images in different patterns, HSV and Dithered images are giving 100\% accuracy in correct retrieval when DCT transform technique is used but the number of images retrieved are less than DWT. In DWT, the response is average for all the formats and more images are retrieved than DCT.

\subsubsection{Accuracy Rate:}

It is important to know the retrieved rate of correct images, means that out of how many correct images available in the database, the correct number of images are retrieved. It will give the clear view of rate of correctness in the retrieval.

Accuracy Rate $=\frac{\text { No.of correct } \text { images retrieved }}{\text { No.of correct } \text { images available }}$

The table below is showing Rate of Accuracy in correct retrieval for all the four formats using the three techniques i.e. GLCM, DWT and DCT

Accuracy in correct retrieval= No. of correct images retrieved

No.of total images retrieved 
Table 10. Accuracy Rate when used GLCM computation

\begin{tabular}{|c|c|c|}
\hline FORMAT & $\begin{array}{c}\text { Rate of Accuracy } \\
\text { for DWT }\end{array}$ & $\begin{array}{c}\text { Rate of Accuracy } \\
\text { for DCT }\end{array}$ \\
\hline RGB & $40 \%$ & $20 \%$ \\
\hline HSV & $80 \%$ & $100 \%$ \\
\hline YIQ & $60 \%$ & $55 \%$ \\
\hline Dithered & $60 \%$ & $30 \%$ \\
\hline \multicolumn{3}{|c|}{ Graph2. Accuracy Rate } \\
\hline $\begin{array}{r}120 \% \\
100 \% \\
80 \% \\
60 \% \\
40 \% \\
20 \% \\
0 \%\end{array}$ & $e^{-1}+n^{e^{e^{e}}}$ & $\begin{array}{l}\text { Rate of } \\
\text { Accuracy } \\
\text { for DWT } \\
\text { Rate of } \\
\text { Accuracy } \\
\text { for DCT }\end{array}$ \\
\hline
\end{tabular}

From the above graph, the clear picture of retrieving techniques along with the formats can be seen. The graph is showing that DCT is best suited when HSV images are to be retrieved. As for other formats, DCT is not showing good performance. DWT is $80 \%$ for HSV and less for all other formats.

\section{CONCLUSION:}

As the demand of retrieval of similar images is increasing in internet day by day. A proper algorithm should be there to resolve this. This can only be done if it is known that which format is best suited through which retrieval technique. Here in this research, it is concluded that whenever HSV images are to be retrieved, DCT transform is best suited. But for other formats, DWT can be used as it is giving above average performance in correct retrieval. In future, other techniques like PCA, Gabor filters, etc can also be used for retrieving the images.

\section{REFERENCES}

[1] Jane, Tharam Dillon, Edwige Pissaloux, "Feature Guide: Statistically based Feature Selection Scheme", IEEE, PP.717-720, 2001.
[2] YUAN Hai Ying, The Image Compression Research on the Preprocessing Technology of Lifting Wavelet Transform", International Conference on Computer Technology and Development, IEEE pp 637 640May 2010.

[3] Rukun Hu1, Shuai Shao, Ping Guo, "Investigating Visual Feature Extraction Methods for Image Annotation", IEEE International Conference on Systems, Man, and Cybernetics Vol.2, No.1,pp. 3122 - 3127, October 2009.

[4] Samia G. Omar, Mohamed A. Ismail and Sahar M. Ghanem, "WAY-LOOK4: A CBIR System Based on Class Signature of the Images' Color and Texture Features", Computer Systems and Applications, Vol. 14, No. 2, pp.464 - 471, Aug. 2009.

[5] Ryszard S. Chora's, "Image Feature Extraction Techniques and Their Applications for CBIR and Biometrics Systems", BIOLOGY AND BIOMEDICAL ENGINEERING, Vol.1, pp. 6 - 16, 1, 2007.

[6] Andrzej Materka and Michal Strzelecki,“ Texture Analysis Methods - A Review”, COST B11 report, Brussels, pp 1-33,1998.

[7] Dr. H.B.Kekre, Sudeep D. Thepade, Tanuja K. Sarode and Vashali Suryawanshi, "Image Retrieval using Texture Features extracted from GLCM, LBG and KPE", International Journal of Computer Theory and Engineering, Vol. 2, No. 5, pp 695 700, October, 2010.

[8] Ramadass Sudhir, Lt. Dr. S. Santhosh Baboo, "An Efficient CBIR Technique with YUV Color Space and Texture Features", Computer Engineering and Intelligent Systems, Vol 2, No.6, pp 78 - 85, 2011.

[9] Teinwei Tsai, Yo- ping Huang, Te- Wei Chiang, "Image Retrieval Based on Dominant Texture Features", IEEE ISIE, pp 441- 446, July 9-12, 2006.

[10] Bart M. ter Haar Romeny, "Dither Removal", The Mathematica Journal,Vol. 10, pp 432-441, 2006.

[11] B.V. Ramana Reddy, A. Suresh, M. Radhika Mani, and V.Vijaya Kumar, "Classification of Textures Based on Features Extracted from Pre-processing Images on Random Windows", International Journal of Advanced Science and Technology, Volume 9, pp 9-18, August, 2009.

[12] Golam Sorwar, Ajith Abrah, "Dct based Texture Classification using Soft Computing Approach".

[13] Hafiz Imtiaz, Shaikh Anowarul Fattah, "A DCTbased Local Feature Extraction Algorithm for Palmprint Recognition", International Journal of Scientific \& Technology Research Volume 1, Issue 2, pp1-8, March 2012. 